\title{
THE PRIMARY TARGET FACILITY FOR A NEUTRINO FACTORY BASED ON MUON BEAMS
}

\author{
A. Hassenein, Argonne National Laboratory, Argonne, IL 60439, USA \\ S.A. Kahn, B.J. King, H.G. Kirk, H. Ludewig, R.B. Palmer, C.E. Pearson, R. Samulyak, N. Simos, \\ I. Stumer, P. Thieberger, R.J. Weggel, BNL, Upton, NY 11973, USA \\ Y. Fukui, UCLA, Los Angeles, CA 90095, USA \\ C.D. Johnson, H. Ravn, CERN, Geneva, Switzerland \\ P. Hwang, G. Naumovitch, Everson Electric Co., Bethlehem, PA 18017, USA \\ S. Childress, N.V. Mokhov, FNAL, Batavia, IL 60510, USA \\ M.A. Green, L.L. Reginato, LBNL, Berkeley, CA 94720, USA \\ A. Zeller, Michigan State University, East Lansing, MI 48824, USA \\ J.R. Miller, NHMFL, Tallahassee, FL 32310, USA \\ C. Lu, K.T. McDonald*, E.J. Prebys, Princeton University, Princeton, NJ 08544, USA \\ T.A. Gabriel, J.R. Haines, P.T. Spampinato, ORNL, Oak Ridge, TN 37831, USA
}

\begin{abstract}
Neutrino beams from the decay of muons in a storage ring offer the prospect of very high flux, well-understood spectra, and equal numbers of electron and muon neutrinos, as desirable for detailed exploration of neutrino oscillations via long baseline detectors [1]. Such beams require large numbers of muons, and hence a high performance target station at which a 1-4 MW proton beam of $16-24 \mathrm{GeV}$ impinges on a compact target, all inside a high field solenoid channel to capture as much of the phase volume of soft pions as possible. A first concept was based on a carbon target, as reported in 2000 the Neutrino Factory Study-I [2]. A higher performance option based on a free mercury jet has been studied in 2001 as part of the Neutrino Factory Feasibility Study-II [3, 4]. An overview of a mercury jet target facility is presented here, including requirements, design concept and summaries of simulated performance. Further details are presented in related papers at this conference.
\end{abstract}

\section{THE TARGET FACILITY}

A muon collider [18] or a neutrino factory based on a muon storage ring $[1,2,3,4]$ require intense beams of muons, which are obtained from the decay of pions produced in proton-nucleus collisions. To maximize the yield, pions of momentum near $300 \mathrm{MeV} / c$ should be captured, as illustrated in Fig. 1. For proton energies above $10 \mathrm{GeV}$, the pion yield per unit of proton beam energy is larger for a high- $Z$ target [5]. For proton beam energies in the MW range, beam heating would melt/boil a stationary high- $Z$ target, so a moving target must be used. A mercury jet target is the main option considered here, although several alternatives remain under active study [6, 7]. For greater detail, consult Chap. 3 of [3]. See also [8].

The low-energy pions are produced with relatively large

*mcdonald@puphep.princeton.edu

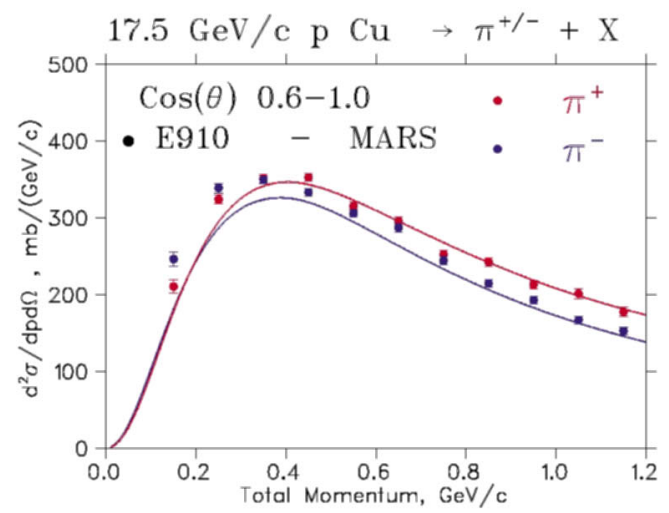

Figure 1: Comparison of pion yield measured in BNL E910 with a MARS calculation.

angles to the proton beam, and efficient capture into a decay and phase rotation channel [9] is obtained by surrounding the target with a 20-T solenoid magnet, whose field tapers down to 1.25 T over several meters, as sketched in Fig. 2. Pion yield is maximized with a mercury target in the form a 1-cm-diameter cylinder, tilted by about $100 \mathrm{mrad}$ with respect to the magnetic axis. To permit the proton beam to interact with the target over 2 interaction lengths, the proton beam is tilted by 33 mrad with respect to the mercury jet axis. See also Fig. 3.

A mercury pool inside the capture solenoid intercepts the mercury jet and the unscattered proton beam, as shown in Fig. 4. The mercury pool, surrounding tungsten carbide/water shielding, and the resistive insert of the 20-T capture magnet [10] are isolated from upstream and downstream beamline elements by a pair of double-walled Be windows. This entire unit can be replaced by remote manipulation should failure occur. The absorbed radiation dose on components near the target is quite large [5], as illustrated in Fig. 5, such that in a $4 \mathrm{Mw}$ proton beam, their 


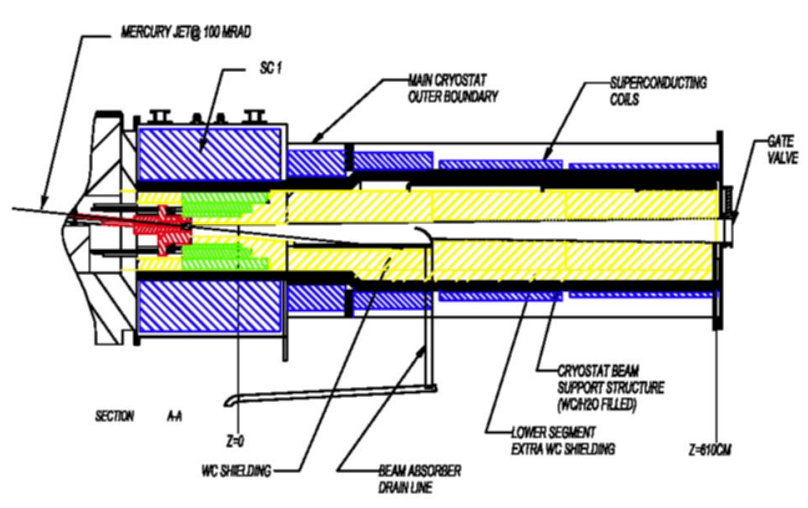

Figure 2: Sketch of the target and capture system based on a mercury jet inside a 20-T solenoid magnet.

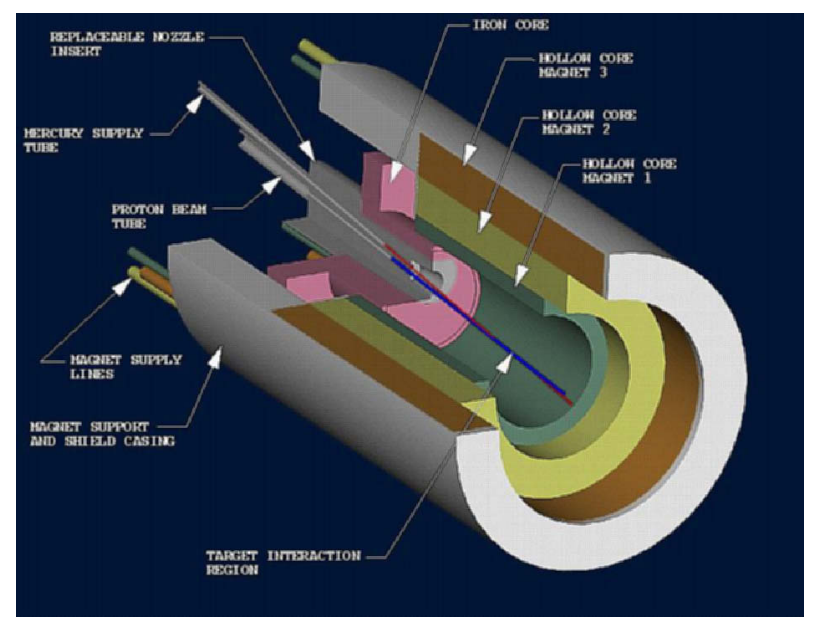

Figure 3: The inner region of the 20-T capture magnet along with the tilted mercury jet target and proton beam.

lifetime against radiation damage may only be 5 years.

The capture solenoid is encased in thick concrete shielding as part of the target facility that includes an overhead crane, hot cells with remote manipulation capability, and a mercury pumping and purification loop [11], as sketched in Fig. 6.

The use of a mercury jet target raises several novel issues. The rapid energy deposition in the mercury target by the proton beam leads to intense pressure waves that can disperse the mercury $[12,13,14]$. Further, as the mercury enters the strong magnetic field eddy currents are induced in the mercury, and the Lorentz force on these currents could lead to distortion of the jet $[15,16]$. On the other hand, the magnetic pressure on the mercury once inside the solenoid will damp mechanical perturbation of the jet.

An $R \& D$ program is underway to assess these critical issues [17].

\section{REFERENCES}

[1] C. Albright et al., Physics at a Neutrino Factory, hep-ex/0008064.

[2] N. Holtkamp, D. Finley, eds., A Feasibility Study of a Neu-
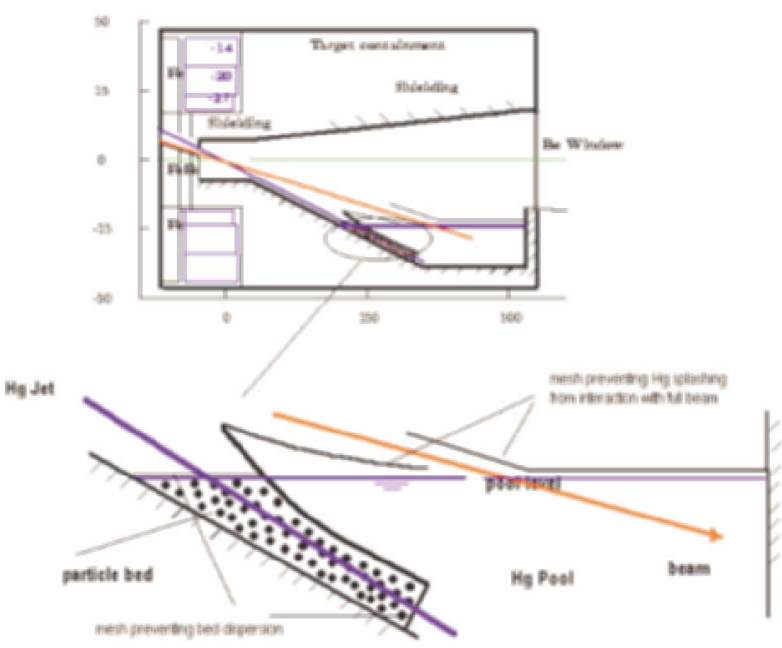

Figure 4: A mercury pool inside the capture solenoid intercepts the mercury jet and the unscattered proton beam.

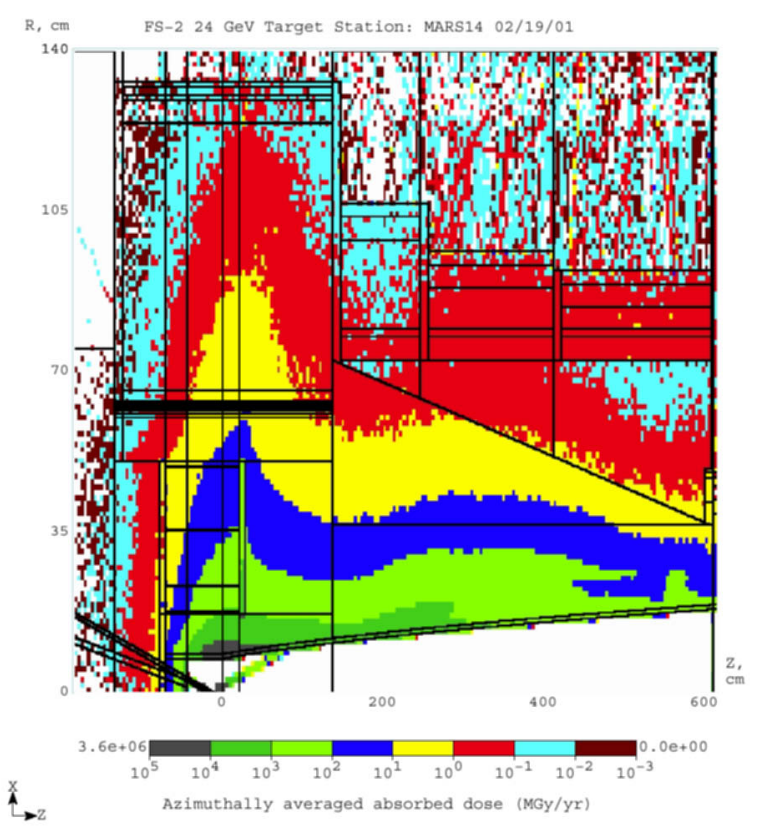

Figure 5: Absorber radiation dose in the pion capture system per $2 \times 10^{7} \mathrm{~s}$ of a $1-\mathrm{MW}, 24-\mathrm{GeV}$ proton beam on a mercury target.

trino Source Based on A Muon Storage Ring, http://www.fnal.gov/projects/muon_collider/nu-factory/

[3] S. Ozaki et al., eds., Feasibility Study-II of a Muon-Based Neutrino Source,

http://www.cap.bnl.gov/mumu/studyii/FS2-report.html

[4] R. Palmer et al., A Second Feasibility Study of a Muon Storage Ring Neutrino Factory, PAC2001, FOAC007.

[5] N. Mokhov, Particle Production and Radiation Environment at a Neutrino Factory Target Station, PAC2001, FOAC010.

[6] B. King et al., An Inconel Rotating Band Pion Production Target for Neutrino Factories and Muon Colliders, PAC2001, TPAH136. 


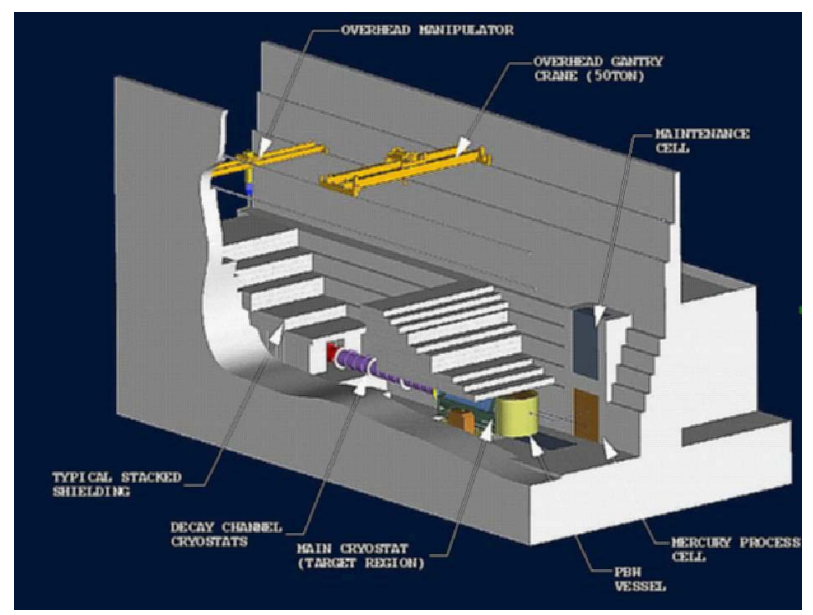

Figure 6: Sketch of the target facility.

[7] V. Balbekov and N. Mokhov, Low-Budget Muon Source, PAC2001, TPAH144.

[8] T. Gabriel et al., Targets for High-Intensity Particle Production, PAC2001, FOAC008.

[9] H. Kirk et al., Phase Rotation at the Front End of a Neutrino Factory, PAC2001, TPPH161.

[10] R. Weggel et al., Design Study for 20 T, $15 \mathrm{~cm}$ Bore Hybrid Magnet with Radiation-Resistant Insert for Pion Capture, PAC2001, RPPH074.

[11] P. Spampinato et al., Target Enclosure/System Design for a Mercury-Target Neutrino Producing Facility, PAC2001, TPAH154.

[12] N. Simos et al., Thermodynamic Interaction of the Primary Proton Beam with a Mercury Jet Target at a Neutrino Factory Source, PAC2001, RPAH076.

[13] A. Hassanein and I. Konkashbaev, Thermoelastic Response of Suddenly Heated Liquid and Solid Targets for High Power Colliders, PAC2001, RPAH071.

[14] R. Samulyak and W. Oh, Global Numerical Modeling of the Muon Collider Target, PAC2001, TPAH139.

[15] S. Kahn et al., Calculations for a Mercury Jet Target in a Solenoid Magnet Capture System, PAC2001, ROPB010.

[16] A. Hassanein and I. Konkashbaev, Dynamics of Liquid Metal Jets Penetrating a Strong Magnetic Field, PAC2001, ROPB009.

[17] K. Brown et al., The R\&D Program for Targetry at a Neutrino Factory, PAC2001, TPAH156.

[18] C.M. Ankenbrandt et al. (The Muon Collider Collaboration), Status of muon collider research and development and future plans, Phys. Rev. ST Accel. Beams 2, 081001 (1999), http://prst-ab.aps.org/abstract/PRSTAB/V2/i8/E081001/ 